\title{
Acidentes Automobilísticos no Brasil em 2017: estudo ecológico dos anos de vida perdidos por incapacidade
}

\author{
Automobile Accidents in Brazil in 2017: ecological study of the \\ disability-adjusted life years
}

Tamires Feitosa de Lima', Raimunda Hermelinda Maia Macena², Rosa Maria Salani Mota ${ }^{\mathbf{3}}$

DOI: 10.1590/0103-1104201912314

RESUMO Este estudo se propõe a descrever o impacto dos Acidentes Automobilísticos no Brasil, no ano de 2017, sobre os anos de vida perdidos ajustados por incapacidade. Estudo ecológico, com dados secundários da Global Health Data Exchange (Global Burden of Disease 2017) disponibilizados pelo Institute for Health Metricsand Evaluation. Os dados foram obtidos utilizando filtros de causa (ferimentos na estrada por veículo automotor), localização (global, Brasil e sul da América Latina) e indivíduos de ambos os sexos, com idades entre 15 e 49 anos. Foram considerados os anos de vida perdidos por incapacidade e a taxa de mortes por 100 mil habitantes. Os dados foram exportados para o Excel $^{\circledR}$ for Windows 2010 e analisados conforme a literatura. Observa-se, no Brasil, um decréscimo nas taxas de óbitos (19,68\%) e de anos de vida perdidos por incapacidade (22,10\%) por acidente automobilístico entre indivíduos de ambos os sexos, com idades entre 15 e 49 anos, porém mostram-se superiores as taxas globais e do sul da América Latina. Estudos como este são importantes para o aperfeiçoamento e o direcionamento de politicas públicas específicas, para formular e implementar estratégias de promoção e prevenção da saúde de segurança no trânsito.

PALAVRAS-CHAVE Carga Global da Doença. Anos de vida ajustados pela incapacidade. Acidentes de trânsito. Automóveis.

ABSTRACT This study aims to describe the impact of Automobile Accidents in Brazil, in the year 2017, on the years of life lost adjusted for disability. Ecological study, with secondary data from the Global Health Data Exchange (Global Burden of Disease 2017) provided by the Institute for Health Metrics and Evaluation (IHME). The data were obtained using cause filters (road injuries by motor vehicle), location (Global, Brazil and Southern Latin America) and individuals of both sexes, aged 15 to 49 years. Rates of death and DisabilityAdjusted Life Years per 100 thousand inhabitants were considered. The data were exported to Excel ${ }^{\circledR}$ for Windows 2010 and analyzed according to the literature. It is observed, in Brazil, a decrease in death rates (19.68\%) and Disability-Adjusted Life Years (22.10\%) per automobile accident among individuals of both sexes, between 15 and 49 years, but they are higher than the Global and Southern rates of Latin America. Studies such as this are important for the improvement and targeting of specific public policies, to formulate and implement strategies for health promotion and prevention of traffic safety. 


\section{Introdução}

Os Acidentes de Trânsito (AT) ocupam, atualmente, devido à sua elevada morbimortalidade, a nona posição entre as principais causas de morte no mundo, representando um grave problema de saúde pública. O Brasil se mantém em terceiro lugar entre os países com maior número de mortes no trânsito, sendo os $\mathrm{AT}$ a segunda causa de morte não natural evitável, gerando em torno de 40 mil óbitos por ano ${ }^{1}$.

Ainda são escassos os estudos sobre Acidentes Automobilísticos (AA), pois persiste uma confusão semântica nos termos nas diversas legislações e na literatura disponível no Brasil2-4. O AT é um evento em que um ou mais indivíduos, na direção de veículo(s) motorizado(s), causa(m) a interferência no tráfego de outros veículos em vias terrestres, podendo gerar danos físicos e/ou materiais. Já o AA é uma das modalidades de AT, sendo conceituado como um acidente que envolve veículo automotor, de quatro rodas, e que pode transportar, no máximo, de oito a dez pessoas, incluindo o condutor ${ }^{2-4}$.

Os AA têm causas multifatoriais (humana, social, socioeconômica, viária, veicular e ambiental) e geram impactos na saúde, na economia e nos aspectos socioeconômicos de uma população $0^{5}$. Os impactos na saúde ultrapassam as questões de morbimortalidade, pois são capazes de gerar incapacidades e perdas na funcionalidade, temporárias ou permanentes. Nos últimos anos, as estratégias de prevenção e políticas públicas em saúde têm sido desenvolvidas e implementadas considerando indicadores de saúde, estatísticas vitais e inquéritos.

Entretanto, esta modalidade de vigilância dos agravos tem sido subestimada em relação ao impacto de um agravo sobre determinada população. O Global Burden of Disease (GBD) ou Estudo da Carga Global de Doenças (CGD) apresenta-se como uma proposta inovadora de indicador complementar comparável de estatísticas de saúde, com o intuito de quantificar a magnitude causada pela perda de saúde em decorrência de doenças e lesões ${ }^{6-8}$. O GBD é capaz de mensurar os anos de vida perdidos por incapacidade (Disability-Adjusted Life Years - Daly), possibilitando a compreensão do número de anos de vida perdidos resultante da morte prematura e acrescido dos anos vividos com invalidez por um determinado agravo $0^{6,8-10}$.

Deste modo, a quantificação do Daly por um determinado agravo proporciona uma maior precisão do impacto deste agravo em uma determinada população, fornecendo, ainda, informações sobre os fatores intervenientes e os grupos vulneráveis ${ }^{6,8,10}$. Pela escassez de estudos sobre GBD em relação aos AA, este estudo se propõe a descrever o impacto dos AA no Brasil, no ano de 2017, sobre os anos de vida perdidos ajustados por incapacidade.

\section{Métodos}

Estudo ecológico, utilizando dados secundários da Global Health Data Exchange (GBD 2017) disponibilizados pelo Institute for Health Metricsand Evaluation (IHME) ${ }^{11}$, através do site http://www.healthdata.org/, coletados entre novembro e dezembro de 2018, por três pesquisadores cegos. Os dados disponíveis para download são livres, anônimos e podem ser usados e compartilhados de acordo com a Licença de Atribuição do Open Data Commons, porém devem ser citados constando data de acesso, bem como o Universal Resource Locator (a URL) (https://vizhub.healthdata.org/gbd-compare/).

Os dados foram obtidos utilizando como filtros: 1. Causa: ferimentos na estrada por veículo automotor; 2. Localização: global, Brasil e sul da América Latina (Argentina, Chile e Uruguai); 3. Indivíduos: ambos os sexos, com idades entre 15 e 49 anos, pois segundo o sistema de Vigilância de Violências e Acidentes de 2014 (Viva 2014) e a Organização Mundial da Saúde (OMS), os indivíduos de 20 a 39 anos e os de 15 a 29 anos, respectivamente, são os principais envolvidos em AT, sendo considerados os principais grupos de risco ${ }^{\mathbf{1 2}}$. Foram consideradas as taxas de mortes e o Daly para 100 mil habitantes. 
Para o cálculo do Daly foram considerados: anos de vida perdidos por mortalidade prematura (Years of Life Lost - YLL) acrescidos dos anos vividos com invalidez (Years Lost Due to Disability - YLD), utilizando o ano de 2017. Tanto o YLL quanto o YLD são calculados baseando-se na esperança de vida ideal, padronizada mundialmente como 80 anos para homens e 82,5 anos para mulheres, sendo considerado um peso, proposto pelo IHME, atribuído à morte, à doença e à sequela ${ }^{6}$.

Foram incluídas no cálculo a função de ponderação de idade, na qual os anos perdidos para crianças e idosos têm pesos menores, e uma taxa de desconto de $3 \%$, que é inserida aos anos de vida perdidos no futuro ${ }^{6,8,13}$. O resultado do Daly se dá mediante a uma escada de saúde com variação de 0 a 1 , sendo 0 a condição de absoluta saúde e 1 a condição de óbito. Este resultado representa a quantidade de anos de vida saudáveis perdidos ajustados por incapacidades, ou seja, um Daly corresponde a um ano de vida perdido ajustado à invalidez para 100 mil habitantes $6,8,13$.
As análises descritivas puderam ser obtidas através das ferramentas GBD Compare/Viz Hub contidas no IHME, sendo estes dados exportados para o Exce $^{\circledR}$ for Windows 2010.

Os dados utilizados no estudo são de banco de dados de domínio público, contudo, este estudo foi também aprovado pelo Comitê de Ética em Pesquisa Unicatólica Quixadá (CE) (Parecer $\mathrm{n}^{0}$ 3.077.751).

\section{Resultados}

Observa-se, no Brasil, um decréscimo nas taxas de óbitos (19,68\%) por AA de indivíduos, em ambos os sexos, entre 15 e 49 anos (11,18 óbitos por 100 mil habitantes para 8,98 óbitos por 100 mil habitantes). No entanto, a mortalidade brasileira por AA mostra-se superior à taxa global (10,01 óbitos por 100 mil habitantes para 6,86 óbitos por 100 mil habitantes) e à do sul da América Latina (7,93 óbitos por 100 mil habitantes para 7,91 óbitos por $100 \mathrm{mil}$ habitantes) (gráfico 1).

Gráfico 1. Taxa de óbitos por 100 mil habitantes decorrentes de acidentes automobilísticos entre indivíduos de ambos os sexos, entre 15 e 49 anos. Global, Brasil e sul da América Latina, para os anos de 1990 a 2017

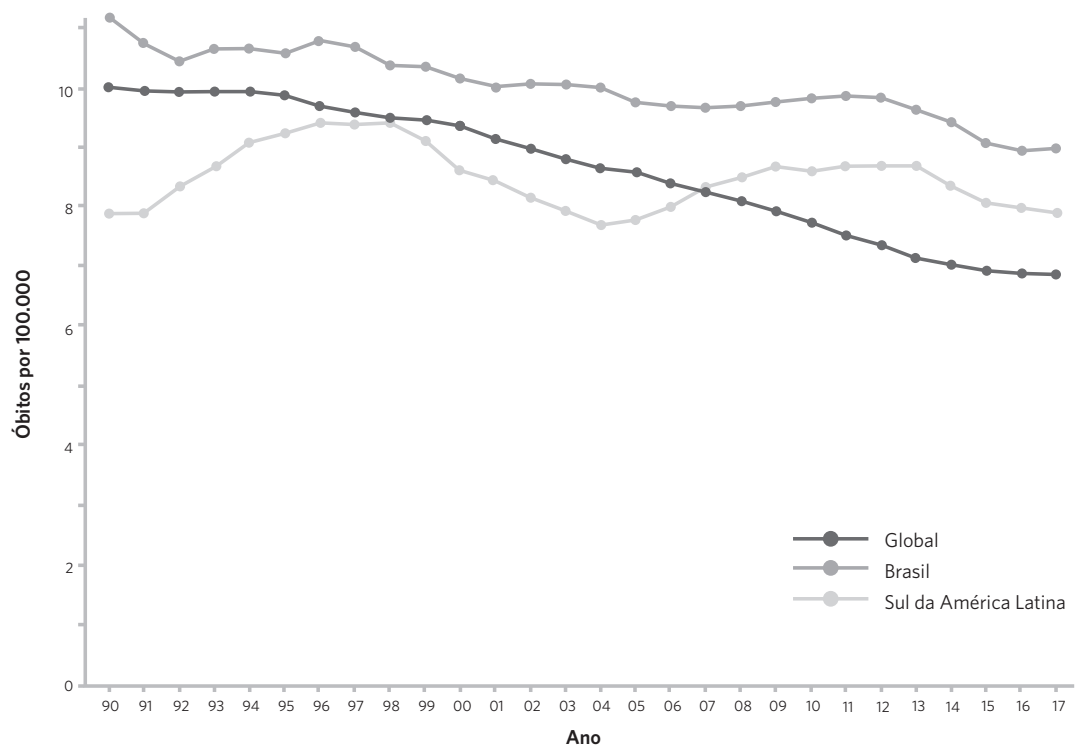

Fonte: http://www.healthdata.org/results/data-visualizations. Data de acesso: 28 de nov. de 2018 11. 
Há, no Brasil, um declínio do Daly (22,10\%) por AA de indivíduos, em ambos os sexos, entre 15 e 49 anos (654,83 Daly por 100 mil habitantes para 510,13 Daly por 100 mil habitantes). Apesar disto, a taxa de Daly por AA é superior à taxa global (614,97 Daly por 100 mil habitantes para 416,91 Daly por 100 mil habitantes) e à do sul da América Latina (479,49 Daly por 100 mil habitantes para 490,51 Daly por $100 \mathrm{mil}$ habitantes) (gráfico 2).

Gráfico 2. Série temporal da taxa de Daly - Disability-Adjusted Life Years (Anos de Vida Perdidos Ajustados por Incapacidade) por 100 mil habitantes decorrente de acidentes automobilísticos entre indivíduos de ambos os sexos, de 15 a 49 anos. Comparação entre a taxa brasileira, a global e a do sul da América Latina, de 1990 a 2017

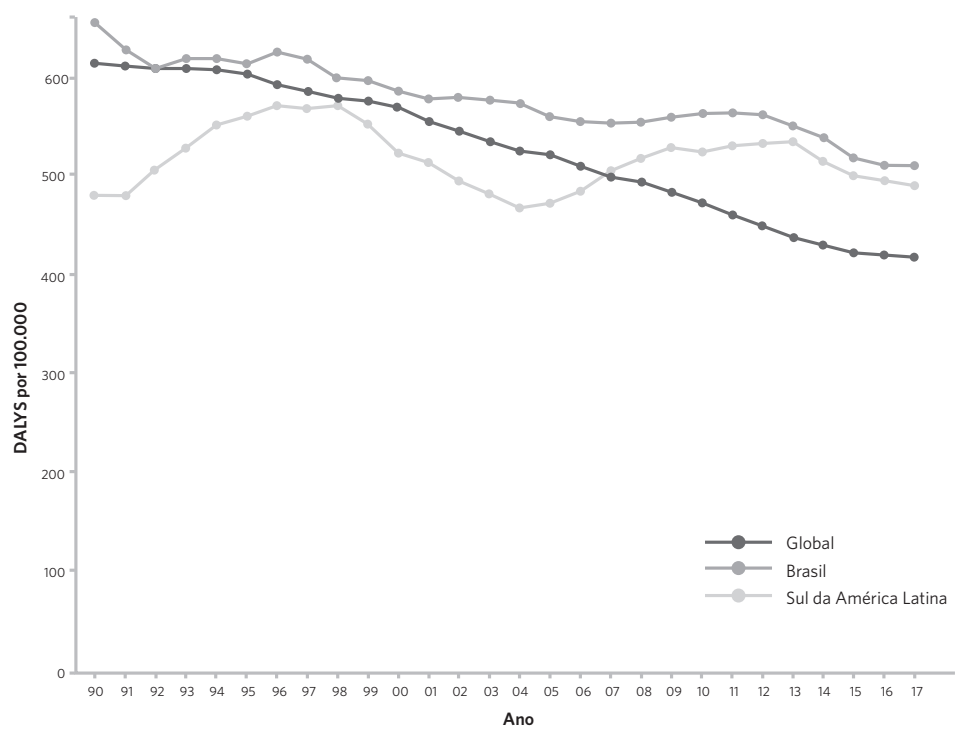

Fonte: http://www.healthdata.org/results/data-visualizations. Data de acesso: 28 de nov. de 2018 11.

Entre os anos de 2007 e 2017, foi observada uma redução na média da taxa de mortalidade por AA entre homens (9,20\%) e um discreto incremento entre as mulheres (6,25\%). Entre os homens, quando comparado a 2007 , no período de 2007 a 2012, a taxa média manteve-se em torno de 15,8 por 100 mil habitantes, com queda crescente nos anos de $2013(1,23 \%), 2014$ (3,68\%), 2015 (7,36\%) e 2016/2017 (9,20\%). Entre as mulheres, quando comparado a 2007, ocorreu incremento das taxas entre 2008 e 2014 (5,36\%), com taxas estáveis e maiores entre 2009 e 2013, o que se repetiu em 2017 (6,25\%) (quadro 1). 
Quadro 1. Taxa de mortalidade e Daly - Disability-Adjusted Life Years (Anos de Vida Perdidos Ajustados por Incapacidade) por 100 mil habitantes, decorrente de acidentes automobilísticos, segundo sexo dos indivíduos, de 15 a 49 anos, no Brasil, de 2007 a 2017

\begin{tabular}{lllllllll}
\hline & \multicolumn{4}{c}{ Sexo Masculino } & \multicolumn{4}{c}{ Sexo Feminino } \\
\cline { 2 - 8 } Ano & \multicolumn{2}{c}{ Mortalidade } & \multicolumn{3}{c}{ DALY } & \multicolumn{1}{c}{ Mortalidade } & DALY \\
\cline { 2 - 8 } & TX & IC & TX & IC $95 \%$ & TX & IC & TX & IC 95\% \\
\hline 2007 & 16,30 & $15,80-16,90$ & 928,80 & $898,60-960,70$ & 3,20 & $3,10-3,30$ & 188,00 & $181,10-195,50$ \\
2008 & 16,30 & $15,80-16,90$ & 927,90 & $897,80-959,10$ & 3,30 & $3,10-3,40$ & 190,40 & $184,00-197,50$ \\
2009 & 16,40 & $15,90-16,90$ & 932,40 & $902,40-962,70$ & 3,40 & $3,20-3,50$ & 196,30 & $189,80-203,00$ \\
2010 & 16,50 & $15,90-17,00$ & 935,40 & $906,20-967,10$ & 3,40 & $3,30-3,50$ & 199,60 & $192,80-206,70$ \\
2011 & 16,50 & $16,00-17,00$ & 935,90 & $908,00-965,90$ & 3,40 & $3,30-3,60$ & 201,00 & $193,80-208,40$ \\
2012 & 16,40 & $15,80-17,00$ & 933,00 & $897,90-964,80$ & 3,40 & $3,30-3,60$ & 200,20 & $192,70-207,70$ \\
2013 & 16,10 & $15,50-16,60$ & 913,20 & $877,10-946,40$ & 3,40 & $3,20-3,50$ & 196,50 & $188,90-204,30$ \\
2014 & 15,70 & $15,10-16,30$ & 892,70 & $856,30-927,30$ & 3,30 & $3,20-3,50$ & 193,70 & $186,10-201,30$ \\
2015 & 15,10 & $14,50-15,60$ & 856,20 & $818,60-889,80$ & 3,20 & $3,10-3,40$ & 188,40 & $180,90-196,00$ \\
2016 & 14,80 & $13,90-15,40$ & 838,20 & $781,70-875,10$ & 3,30 & $3,10-3,40$ & 190,40 & $181,80-198,20$ \\
2017 & 14,80 & $13,30-15,50$ & 833,00 & $752,00-874,60$ & 3,40 & $3,20-3,50$ & 195,50 & $185,00-205,20$ \\
\hline
\end{tabular}

Fonte: http://www.healthdata.org/results/data-visualizations. Data de acesso: 28 de nov. de 201811.

A taxa média de Daly por AA entre homens diminuiu 10,31\% no período de 2007 a 2017, indo de 928,80 para 833,00 anos de vida perdidos ajustados à invalidez por 100 mil habitantes, em 2017, com queda anual progressiva a partir de 2013. Entre as mulheres, a taxa média de Daly cresceu 3,99\% no período de estudo, avançando de 188,00 (2007) para 195,50 anos de vida perdidos ajustados à invalidez por 100 mil habitantes (2017), especialmente entre os anos de 2009 a 2013 (crescimento médio de $5,7 \%$ ) (quadro 1).

\section{Discussões}

A OMS revela que 1,25 milhão de pessoas, por ano, morrem em decorrência de AT, e, aproximadamente, 50 milhões de pessoas sofrem lesões não fatais. Deste modo, o AT é considerado a primeira causa de morte entre adultos jovens, com idades entre $15 \mathrm{e}$ 29 anos, e a terceira causa na faixa etária de 30 a 44 anos $^{1,14}$. Entretanto, o presente estudo demonstra que, no Brasil, nos últimos dez anos, há uma redução nas taxas de óbitos e de Daly por AA de indivíduos, em ambos os sexos, entre 15 e 49 anos, ainda que ambas as taxas sejam superiores à taxa global e à do sul da América Latina.

Os AT são responsáveis por elevados custos financeiros no setor saúde do Brasil, consequentes aos óbitos, traumas e às incapacidades geradas. Uma pesquisa realizada pelo Instituto de Pesquisa Econômica Aplicada (Ipea) em parceria com a Polícia Rodoviária Federal (PRF) em 2015 mostra que os acidentes envolvendo automóveis apresentaram o maior percentual (75,2\%) entre os AT, em 2014 15 . O atlas da acidentalidade no transporte brasileiro traz que, do período entre 2007 e 2017, os acidentes envolvendo automóveis em rodovias federais policiadas foram os que obtiveram o maior número de óbitos, totalizando $34.946(41,9 \%)^{\mathbf{1 6}}$.

Tendo em vista os impactos dos AA nos âmbitos da saúde, social e financeiro, foi estabelecida, no plano de ações da Agenda 2030 para desenvolvimento sustentável, a meta 
mundial de reduzir o número de mortes e lesões consequentes de AT até 2020. Caso as medidas de enfrentamento e redução não sejam tomadas, os AT poderão configurar, em 2030, a sétima causa de morte no mundo ${ }^{\mathbf{1}, \mathbf{1 4}}$. Além disso, em 2014, os acidentes com óbito obtiveram um custo total de $\mathrm{R} \$ 4,5$ bilhões e são responsáveis por, aproximadamente, $35 \%$ dos custos totais com acidentes no País ${ }^{15}$. Em 2015 , os óbitos por AT custaram cerca de R $\$$ 11,6 bilhões e o tratamento das vítimas feridas custou, aproximadamente, $\mathrm{R} \$ 7,7$ bilhões para a economia brasileira17. Neste cenário, os AA necessitam ter sobre si um olhar mais aprimorado, pois apesar de não serem a primeira causa entre os AT, vêm apresentando índices crescentes. Entre os tipos existentes, os motorizados são os mais adquiridos no mercado de vendas de veículos e têm a maior frota entre as diversas modalidades de veículos ${ }^{\mathbf{1 6}, 18}$.

No Brasil, os dados do Viva de 2013 e 2014 mostram que indivíduos do sexo masculino são os que mais se envolvem em AT. Entre os atendidos em serviços sentinelas de urgência e emergência em decorrência de AT, 71,1\% são homens; destes, $8,5 \%$ usaram automóveis como meio de transporte e $38,3 \%$ afirmaram que o outro veículo envolvido no acidente era um automóvel11. O presente estudo demonstra que os homens brasileiros possuem taxas de Daly e óbitos por AA mais elevadas do que as mulheres, porém com tendência a redução ao longo dos anos, em oposição ao que ocorre entre as taxas das mulheres, que apresentam tendência ao crescimento. Há que se destacar que a redução da mortalidade e a melhoria do Daly podem estar associadas à principal medida de enfrentamento da violência no trânsito implantada no Brasil, que foi a instituição do Código de Trânsito Brasileiro (CTB), em 19982,19,20. O CTB estabeleceu atribuições de órgãos de trânsito, normas, condutas, infrações e penalidades aos condutores ${ }^{\mathbf{2}, 21}$. Outro elemento importante foi a Lei Seca, implementada em 2008 pelo governo brasileiro, que estabeleceu penalidades para condutores que estivessem sob efeito de álcool, com o intuito de minimizar/eliminar um dos principais fatores causadores dos $\mathrm{AT}^{\mathbf{2 1}}$.

Há que se destacar que nem sempre um AA gera óbito. Devido à maior proteção que o automóvel oferece ao indivíduo, a gravidade dos AA é menor quando comparada aos acidentes motociclísticos, contudo, os envolvidos nos eventos apresentam sequelas e incapacidades físicas e/ou mentais ${ }^{22}$. Um estudo de carga global de doença realizado no Brasil mostra, em um comparativo entre os anos de 1990 e 2015, que os AT são, entre os eventos de causa externa, os que apresentam maior taxa de Daly por 100 mil habitantes no País, sendo que, em 1990, os acidentes de veículo motorizados ocupavam o terceiro lugar e, em 2015, passaram a assumir a quarta colocação 23 .

O Brasil tem como medida de enfrentamento e controle de AT a legislação vigente e políticas públicas voltadas para o agravo. Além disto, investe em estratégias educacionais através das mídias sociais para a prevenção de sua ocorrência e melhorias na infraestrutura rodoviária do País. A Política Nacional de Redução da Morbimortalidade por Acidentes e Violência e a Política Nacional de Promoção à Saúde fazem parte da Rede Nacional de Prevenção de Violência e Promoção da Saúde e representam as principais políticas de saúde direcionadas à prevenção e promoção da saúde e cultura de paz no Brasil21,24.

Apesar disso, os dados do presente estudo sinalizam que os homens estão vivendo menos anos com incapacidades do que as mulheres, o que pode ser explicado pelos estereótipos e valores no trânsito presentes no imaginário popular, de que homens são imprudentes e ameaçadores para o trânsito, porém mais seguros ao volante. $\mathrm{O}$ fato de as mulheres serem vistas como prudentes, obedientes e cautelosas pode explicar porque elas vivem mais anos com incapacidade por seus acidentes, que tendem a ter menor gravidade ${ }^{19,25}$.

As limitações deste estudo são as próprias do modelo ecológico. Inicialmente, os aspectos verificados na coletividade dos AA podem não refletir a real condição individual dos cidadãos 
brasileiros (falácia ecológica), tendo em vista que as taxas encontradas para esse evento estiveram relacionadas a um grupo populacional específico (15 a 49 anos). A unidade de análise constituiu-se dos Daly por AA, de maneira a ser impossível inferir se os indivíduos que tiveram acidentes menos graves tiveram melhor desempenho no Daly. Outras limitações deste estudo referem-se às características do Sistema de Informações de Mortalidade (SIM), que é o principal fornecedor de dados ao IHME, além de não ser possível determinar as duplas e triplas entradas de usuários no sistema (readmissões). Acrescentam-se, ainda, fatores como o registro ou o sub-registro. Entretanto, o SIM é um sistema oficial, e diversos estudos apontam a validade e a utilidade dos seus dados, garantindo, assim, a consistência dos que foram apresenta$\operatorname{dos}^{\mathbf{8}, \mathbf{9}, 23}$. Estudos como este, que abordam os impactos do AA, são importantes para o aperfeiçoamento e o direcionamento de politicas públicas específicas, para formular e implementar estratégias de promoção e prevenção da saúde de segurança no trânsito.

\section{Conclusões}

Há redução na mortalidade e nos anos de vida perdidos ajustados à incapacidade por
AA no Brasil, contudo, a mortalidade e o Daly ainda apresentam taxas superiores, se comparados a outros países do mundo e ao sul da América Latina.

Faz-se necessário o desenvolvimento de estudos não ecológicos para analisar a tendência e o público mais vulnerável ao AA no Brasil, o que permitirá o norteamento de políticas públicas efetivas. A baixa visibilidade feminina, pelas políticas e estratégias de controle e redução dos AA, carece ser ponderada e novas medidas devem ser implementadas, com o intuito de reduzir os anos de vida vividos com incapacidades.

\section{Colaboradores}

Lima TF (0000-0002-3300-2366)* contribuiu para a concepção do trabalho, elaboração crítica do conteúdo intelectual, análise e interpretação de dados e aprovação da versão final do manuscrito. Macena RHM (0000-00023320-8380)* contribuiu para a concepção, o planejamento, a análise e a interpretação dos dados, a revisão crítica do conteúdo e a aprovação da versão final do manuscrito. Mota RMS (0000-0002-3347-8372)* contribuiu para a revisão crítica do conteúdo e a aprovação da versão final. 


\section{Referências}

1. World Health Organization. Global Status Report on Road Safety. Geneva: WHO; 2015.

2. Brasil. Lei no 9.503, de 23 de Setembro de 1997. 1997 [acesso em 2018 out 2]. Disponível em: http://www. planalto.gov.br/Ccivil_03/leis/L9503Compilado.htm.

3. Associação Brasileira de Normas Técnicas. NBR 10.697: Pesquisa de acidentes de trânsito - terminologia. Rio de Janeiro: ABNT; 1989.

4. Organização Mundial da Saúde. CID-10 - Classificação Estatística Internacional de Doenças e Problemas Relacionados à Saúde. Geneva: OMS; 2008. [acesso em 2018 out 2]. Disponível em: http://www.datasus. gov.br/cid10/V2008/WebHelp/v01_v99.htm.

5. World Health Organization. World report on road traffic injury prevention. Geneva: WHO; 2004.

6. Institute for Health Metricsand Evaluation. Estudo de Carga de Doença Global: gerando evidências, informando políticas de saúde. Seattle, WA: IHME; 2013 [acesso em 2018 nov 28]. Disponível em: http:// www.healthdata.org/gbd.

7. Rocha E. A carga global de doença: fonte de informação para a definição de políticas e avaliação de intervenções em saúde. Rev. Port. Cardiol. 2017; 36(4):283285.

8. Revista Brasileira de Epidemiologia. Estudo de carga global de doença 2015: resumo dos métodos utilizados. Rev. bras. Epidemiol. 2017; 20(1):4-20.

9. Souza MFM, França EB, Cavalcante A. Carga da doença e análise da situação de saúde: resultados da rede de trabalho do Global Burden of Disease (GBD) Brasil. Rev. Bras. Epidemiol. 2017; 20(1):1-3.

10. Haagsma JA, Graetz N, Bolliger I, et al. The global burden of injury: Incidence, mortality, disability-adjusted life years and time trends from the Global Burden of Disease study 2013. Inj. Prev. 2015; 22(1): 3-18.
11. Institute for Health Metricsand Evaluation: measuring what matters. [internet]. Seattle: IHME; 2019. [acesso em 2018 nov 29]. Disponível em: http://www. healthdata.org/.

12. Brasil. Ministério da Saúde, Secretaria de Vigilância em Saúde, Departamento de Vigilância de Doenças e Agravos Não Transmissíveis e Promoção da Saúde. Viva: Vigilância de Violências e Acidentes: 2013 e 2014. Brasília, DF: Ministério da Saúde; 2017.

13. Murray C, Lopez A. The global burden of disease: a comprehensive assessment of mortality and disability from diseases, injuries and risk factors in 1990 and projected to 2020. Cambridge: Harvard University Press; 1996.

14. Organização Pan-Americana da Saúde. Salvar VIDAS - Pacote de medidas técnicas para a segurança no trânsito. In: Organização Mundial da Saúde, editor. Brasília, DF: OPAS; 2018.

15. Instituto de Pesquisa Econômica Aplicada e Polícia Rodoviária Federal. Acidentes de trânsito nas rodovias federais brasileiras: caracterização, tendências e custos para a sociedade. Brasília, DF: IPEA; 2015.

16. Atlas da Acidentalidade no transporte brasileiro. Programa Volvo de Segurança no Trânsito. Curitiba: PVST; 2018. [acesso em 2018 nov 20]. Disponível em: https://www.atlasacidentesnotransporte.com.br/ home.

17. Ambev. Retrato da segurança viária no Brasil 2017. 2017. [acesso em 2018 nov 20]. Disponível em: https:// www.ambev.com.br/conteudo/uploads/2017/09/ Retrato-da-Seguran\%C3\%A7a-Vi\%C3\%Alria_Ambev_2017.pdf.

18. Confederação Nacional do Transporte. Acidentes rodoviários e infraestrutura. Brasília, DF: CNT; 2018.

19. Davantel PP, Pelloso SM, Carvalho MDDB, et al. A mulher e o acidente de trânsito: caracterização do 
evento em Maringá, Paraná. Rev. Bras. Epidemiol. 2009; 12:355-367.

20. Abreu DROM, Souza EM, Mathias TAF. Impacto do Código de Trânsito Brasileiro e da Lei Seca na mortalidade por acidentes de trânsito. Cad. Saúde Pública (Online). 2018; 34(8):1-13.

21. Bacchieri G, Barros AJ. Acidentes de trânsito no Brasil de 1998 a 2010: muitas mudanças e poucos resultados. Rev. Saúde Pública. 2011; 45(5): 949-963.

22. Andrade SSCA, Jorge MHPM. Estimativa de sequelas físicas em vítimas de acidentes de transporte terrestre internadas em hospitais do Sistema Único de Saúde. Rev. Bras. Epidemiol. 2016; 19(1):100-111.

23. Ladeira RM, Malta DC, Morais Neto OL, et al. Aci- dentes de transporte terrestre: estudo Carga Global de Doenças, Brasil e unidades federadas, 1990 e 2015. Rev. Bras. Epidemiol. 2017; 20(1):157-170.

24. Silva Júnior JB, Malta DC. Avaliação de políticas e ações voltadas a prevenção de acidentes de trânsito e violências no Brasil. Epidemiol. Serv. Saúde. 2013; 22(3):371-372.

25. Almeida NDV, Lima AKB, Albuquerque CM, et al. As relações de gênero e as percepções dos/das motoristas no âmbito do sistema de trânsito. Psicol. Ciênc. Prof. 2005; 25:172-185.

Recebido em 03/04/2019

Aprovado em 16/10/2019

Conflito de interesses: inexistente

Suporte financeiro: Fundação Cearense de Apoio ao

Desenvolvimento Científico e Tecnológico (Funcap) 